The Astrophysical JouRnal, 501:375-385, 1998 July 1

(C) 1998. The American Astronomical Society. All rights reserved. Printed in U.S.A.

\title{
A GENERALIZED MODEL FOR THE PROTON EXPANSION IN ASTROPHYSICAL WINDS. II. THE ASSOCIATED SET OF TRANSPORT EQUATIONS
}

\author{
François Leblanc AND DaNiel Hubert \\ Département de Recherche Spatiale, CNRS URA 264, Observatoire de Paris, 92195 Meudon Cedex, France; fleblanc@megasx.obspm.fr \\ Received 1997 February 21 ; accepted 1998 February 4
}

\begin{abstract}
In Paper I, we presented a new model of the velocity distribution function for protons composing a stellar atmosphere expanding in interstellar space, valid from collisional to collisionless regions. In this paper, the set of generalized transport equations associated with this model and the closure assumptions for higher order velocity moments are provided for 9 and 16 moment approximations. The study of the properties of such a set of transport equations in the collisionless limit is presented and discussed. A comparison with the similar bi-Maxwellian approximation is made using two kinds of analysis, in the context of an application to solar wind expansion. Our model is better adapted to high values of the heat flux and thus is able to provide a macroscopic parameter evolution for stellar atmosphere expansion in a state far from local equilibrium, as well as for the expansion of planetary polar winds.
\end{abstract}

Subject headings: hydrodynamics — plasmas — solar wind — stars: atmospheres — stars: mass loss

\section{INTRODUCTION}

In a previous article (Leblanc \& Hubert 1997, hereafter Paper I), we have presented a new model for the proton velocity distribution function (VDF) in astrophysical winds. This function is a polynomial expansion based on an exact solution of the BGK equation for moderately ionized plasmas, and has been constructed following the generalized Grad method (Grad 1958; Mintzer 1965). We have emphasized that the main advantage of this function is that it is close to the observed proton VDF profiles in the solar wind. Indeed, our zeroth-order approximation is an asymmetric function that displays a suprathermal tail in the magnetic field direction. In this paper, we present the transport equations, the moment closure, and the onedimensional derived system associated with our generalized model of the VDF. This approach has been developed to be well adapted to the description of particle states from collisional to collisionless regions (corresponding to large Knudsen numbers and suprathermal velocity distribution functions with large normalized heat flux). Moreover, the moment approach of Grad enables us to derive direct relations between the macroscopic and microscopic descriptions of the particles and between the kinetic effects and the classic hydrodynamic parameters.

In transitional collisional regions, the characteristics of the distribution functions that compose the solar wind are now better known from the solar corona to interplanetary space, thanks to numerous probes like Ulysses and $S O H O$, which provide new information from the solar corona and solar wind (Feldman et al. 1996). But several very important phenomena still need to be accurately understood, e.g., the heating and increase of wind speed in the corona, the nonadiabatic evolution of particles between 0.3 and $1 \mathrm{AU}$ (Marsch et al. 1982; Kohl, Strachan, \& Gardner 1996), the origin of the proton temperature anisotropy (Marsch et al. 1982), the importance of heat flux in the corona (Withbroe 1988), and the interaction between the heavy and light ions (Habbal 1996). Improving the heat-flux conduction law is also important for obtaining a good description of the thermal forces in the corona and interplanetary medium, where the classical Spitzer law is no longer valid (Dorelli \& Scudder 1996). In addition to the wave-particle effects in all these processes (Jacques 1977; Hu, Esser, \& Habbal 1997), we need an accurate description of the Coulomb collisional effects.

In this paper, we establish the new transport equations associated with our generalized velocity distribution function model. We study the fundamental properties generated by the new closure assumption of the equation system, using two approaches that have already been applied in analyzing the bi-Maxwellian model of Demars \& Schunk (Cordier 1994a, 1994b; Levermore, Morokoff, \& Nadiga 1995; Levermore 1995). The hyperbolicity criterion of Cordier and the realizability criterion of Levermore et al. allow us to underline the improvements to the generalized model in the context of measurements made in the solar wind.

Section 2 is devoted to the presentation of the VDF and the macroscopic parameters used to describe the plasma; we then present the closure assumption for higher order moments and the transport equations. Section 3 deals with the properties of the generalized system. In $\S 4$ we discuss the main advantages of the generalized multimoment equations compared to the set of transport equations associated with a bi-Maxwellian function. A conclusion is given in $\S 5$.

\section{THE GENERALIZED TRANSPORT EQUATION SYSTEM}

\subsection{The Velocity Distribution Function}

To describe each species in a gas mixture, we use a separate VDF, $f_{s}\left(\boldsymbol{r}, \boldsymbol{v}_{s}, t\right)$, that is a solution of the Boltzmann equation, where $\boldsymbol{r}$ is the position vector, $\boldsymbol{v}_{s}$ is the species velocity, and $t$ is the time. The distribution function $f_{s}$ can be viewed as a probability density in $\boldsymbol{r}, \boldsymbol{v}_{s}$ phase space. For most flow situations, mathematical difficulties do not allow us to obtain closed-form solutions to Boltzmann's equation. Therefore, these approaches are restricted to the determination of a limited 
number of low-order velocity moments of the species distribution function, which are defined as

$$
\left\langle C_{s}^{p}\right\rangle=\frac{1}{m_{s}} \int_{V} f_{s}(r, v, t) C_{s}^{p} d c_{s},
$$

where $p$ is the order of the velocity moment, $\boldsymbol{c}_{s}=\boldsymbol{v}_{s}-\boldsymbol{u}_{s}$ is the random velocity, $\boldsymbol{u}_{s}$ is the species average drift velocity, $C_{s}^{p}$ is a product of $p$ terms of components $\left(c_{s 1}, c_{s 2}, c_{s 3}\right)$, and $V$ is the velocity space.

The first step in this type of approach consists of choosing an a priori form for $f_{s}$, that is, the type of dependence of the microscopic description $f_{s}$ as a function of the velocity moments. Indeed, to close the transport equations of the generalized state variables, such an approximate expression is needed. As in Demars \& Schunk (1979), we construct this function as a polynomial expansion based on a zeroth-order function (Paper I). In a 16 moment approximation, the macroscopic parameters used to describe the plasma flows derived from the velocity moments are:

$\boldsymbol{u}_{s}=\left\langle\boldsymbol{v}_{s}\right\rangle$
$T_{s \|}=\left(m_{s} / k_{\mathrm{B}}\right)\left\langle c_{s \|}^{2}\right\rangle$
$T_{s \perp}=\left(m_{s} / 2 k_{\mathrm{B}}\right)\left\langle c_{s \perp}^{2}\right\rangle$
$\boldsymbol{q}_{s}^{\|}=n_{s} m_{s}\left\langle c_{s \|}^{2} \boldsymbol{c}_{s}\right\rangle$
$\boldsymbol{q}_{s}^{\perp}=\frac{1}{2} n_{s} m_{s}\left\langle c_{s \perp}^{2} \boldsymbol{c}_{s}\right\rangle$
$\mathbf{P}_{s}=n_{s} m_{s}\left\langle\boldsymbol{c}_{s} \boldsymbol{c}_{s}\right\rangle$
$\tau_{s}=\mathbf{P}_{s}-p_{s \perp} \mathbf{I}-\left(p_{s \|}-p_{s \perp}\right) \boldsymbol{e}_{3} \boldsymbol{e}_{3}$
$\boldsymbol{\mu}_{s}^{\|}=n_{s} m_{s}\left\langle c_{s \|}^{2} \boldsymbol{c}_{s} \boldsymbol{c}_{s}\right\rangle$
$\boldsymbol{\mu}_{s}^{\perp}=n_{s} m_{s}\left\langle c_{s \perp}^{2} \boldsymbol{c}_{s} \boldsymbol{c}_{s}\right\rangle$
$\mathbf{Q}_{s}=n_{s} m_{s}\left\langle\boldsymbol{c}_{s} \boldsymbol{c}_{s} \boldsymbol{c}_{s}\right\rangle$
$p_{s \|}=n_{s} k_{\mathrm{B}} T_{s \|}$
$p_{s \perp}=n_{s} k_{\mathrm{B}} T_{s \perp}$
$T_{s}=\frac{1}{3}\left(T_{s \|}+2 T_{s \perp}\right)=\left(m_{s} / 3 k_{\mathrm{B}}\right)\left\langle c_{s}^{2}\right\rangle$
$\boldsymbol{q}_{s}=\frac{1}{2}\left(\boldsymbol{q}_{s}^{\|\|}+2 \boldsymbol{q}_{s}^{\perp}\right)=\frac{1}{2} \rho_{s}\left\langle c_{s}^{2} \boldsymbol{c}_{s}\right\rangle$

Drift velocity

Parallel temperature

Perpendicular temperature

Heat-flux vector for parallel energy

Heat-flux vector for perpendicular energy

Pressure tensor

Stress tensor

Higher order pressure tensor related to parallel energy

Higher order pressure tensor related to perpendicular energy

Heat-flux tensor, where $n_{s}$ is the number density of the species $s, m_{s}$ is the mass, $k_{\mathrm{B}}$ is Boltzmann's constant, and $\rho_{s}=n_{s} m_{s}$ is the mass density

Parallel energy

Perpendicular energy

Absolute temperature of species $s$

$\boldsymbol{q}_{s}=\frac{1}{2}\left(\boldsymbol{q}_{s}^{\Pi \|}+2 \boldsymbol{q}_{s}^{\perp}\right)=\frac{1}{2} \rho_{s}\left\langle c_{s}^{2} \boldsymbol{c}_{s}\right\rangle \quad$ Heat-flux vector.

The unknowns used in a 16 moment approximation are $n_{s}, \boldsymbol{u}_{s}, T_{s \|}, T_{s \perp}, \tau_{s}, \boldsymbol{q}_{s}^{\|}$, and $\boldsymbol{q}_{s}^{\perp}$. The higher order velocity moments $\boldsymbol{\mu}_{s}^{\|}, \boldsymbol{\mu}_{s}^{\perp}$, and $\mathbf{Q}_{s}$ are expressed starting from the approximate VDF as a function of these parameters. We choose the same convention as in Demars \& Schunk (1979). The subscripts $\|$ and $\perp$ denote quantities related to the parallel and perpendicular directions to the magnetic field, respectively. Thus, for a vector $\boldsymbol{A}=\left(A_{1}, A_{2}, A_{3}\right)^{\top}, \boldsymbol{A}_{\perp}=\left(A_{1}, A_{2}, 0\right)^{\top}$ and $\boldsymbol{A}_{\|}=\left(0,0, A_{3}\right)^{\top}(\mathrm{a}$ superscript $\mathrm{T}$ on a tensor means the elements of the tensor should be transposed). But when the symbols $\|$ and $\perp$ are superscript, the vector $\boldsymbol{A}^{\perp}$ is defined as $\left(A_{1}^{\perp}, A_{2}^{\perp}, A_{3}^{\perp}\right)^{\top}$ and $\boldsymbol{A}_{\|}^{\perp}$ is defined as $\left(0,0, A_{3}^{\perp}\right)^{\top}$, vector $\boldsymbol{A}^{\perp}$ being related to the perpendicular thermal energy (see the definition of $q_{s}^{\|}, q_{s}^{\perp}, \mu_{s}^{\|}$, and $\mu_{s}^{\perp}$ ). The unit dyadic I is defined as $I=e_{1} e_{1}+e_{2} e_{2}+e_{3} e_{3}$ where $\left(e_{1}, e_{2}, e_{3}\right)$ is an orthogonal set of unit vectors, with $e_{3}$ aligned along the direction of the straight magnetic field lines [corresponding to the letter $z$ in the position vector $\left.\boldsymbol{r}=(x, y, z)^{\top}\right]$.

To obtain the 16 moment set of transport equations, we multiply Boltzmann's equation by $m_{s}, m_{s} c_{s}, m_{s} c_{s \|}^{2}, m_{s} c_{s \perp}^{2} / 2, m_{s} c_{s} c_{s}$, $m_{s} c_{s \|}^{2} c_{s}$, and $m_{s} c_{s \perp}^{2} c_{s}$, and integrate over velocity space. We then obtain the continuity, momentum, parallel energy, perpendicular energy, pressure tensor, parallel and perpendicular heat-flux equations, respectively, for species $s$. To close such a system, if we neglect the collisional terms, we only have to express higher order moments $\boldsymbol{\mu}_{s}^{\|}, \boldsymbol{\mu}_{s}^{\perp}$, and $\mathbf{Q}_{s}$ as a function of the lower order velocity moments. But if we do not neglect the collisional terms, we also need an a priori form of the distribution function to determine the collisional terms for the non-Maxwellian interacting potential. To this end, we use the $\operatorname{VDF} f_{S G}^{A}$, defined in Paper I as

$$
f_{s G}^{A}=f_{s G}^{0}\left\{1+\phi_{s}\right\}
$$

and

$$
f_{s G}^{0}\left(\boldsymbol{r}, c_{s \|}, c_{s \perp}, t\right)=n_{s} \frac{m_{s}}{4 k_{\mathrm{B}} \pi T_{s \perp} D_{s}^{*}} \exp \left(-\frac{m_{s}}{2 k_{\mathrm{B}} T_{s \perp}} c_{s \perp}^{2}-\frac{c_{s \|}+D_{s}^{*}}{D_{s}^{*}}+\frac{1}{E_{s}^{*}}\right) \operatorname{erfc}\left[E_{s}^{* 1 / 2}\left(\frac{1}{E_{s}^{*}}-\frac{c_{s \|}+D_{s}^{*}}{2 D_{s}^{*}}\right)\right],
$$

where $c_{s \|}, c_{s \perp}$, and $T_{s \perp}$ have been already defined; erfc is the complementary error function (Abramovitz \& Stegun 1964). $D_{s}^{*}$ and $E_{s}^{*}$ are defined in Paper I. The term $\phi_{s}$ is a polynomial development determined using the rules of orthogonalization defined by Mintzer (1965):

$$
\begin{aligned}
\phi_{s}=\frac{\beta_{s \perp}}{\rho_{s}}\left[\frac{\beta_{s \perp}}{2} \tau_{s}: c_{s \perp} c_{s \perp}+\right. & \left(\beta_{s \|}+\beta_{s 2}^{2}\right) \tau_{s}: c_{s \perp} c_{s \|}+\frac{\beta_{s 2}}{2}\left(\alpha_{s 2} c_{s \|}^{2}+\gamma_{s 2}\right) \tau_{s}: c_{s \perp} e_{3} \\
& \left.+\alpha_{s 2}\left(\gamma_{s 2}+\beta_{s 2} c_{s \|}+\alpha_{s 2} c_{s \|}^{2}\right) q_{s}^{\|} \cdot c_{s \perp}+\beta_{s \perp}\left(\frac{\beta_{s \perp}}{4} c_{s \perp}^{2}-1\right) q_{s}^{\perp} \cdot c_{s \perp}+\beta_{s \|}\left(\frac{\beta_{s \perp}}{2} c_{s \perp}^{2}-1\right) q_{s}^{\perp} \cdot c_{s \|}\right],
\end{aligned}
$$

where

$$
\beta_{s \perp}=\frac{m_{s}}{k_{\mathrm{B}} T_{s \perp}}, \quad \beta_{s \|}=\frac{m_{s}}{k_{\mathrm{B}} T_{s \|}},
$$


and

$$
\alpha_{s 2}=\left[6\left(\frac{q_{s 3}^{\|}}{2 \rho_{s}}\right)^{4 / 3}-\frac{2}{\beta_{s \|}^{2}}-\beta_{s \|}\left(\frac{q_{s 3}^{\|}}{\rho_{s}}\right)^{2}\right]^{-1 / 2}, \quad \beta_{s 2}=-\alpha_{s 2} \frac{q_{s 3}^{\|}}{\rho_{s}} \beta_{s \|}, \quad \gamma_{s 2}=-\frac{\alpha_{s 2}}{\beta_{s \|}} .
$$

The definition of our VDF imposes the condition that the heat flux is limited by the inequality $q_{s 3}^{\|} \leq 2 \rho_{s} w_{s \|}^{3}=2\left(P_{s \|} V_{\text {sth } \|}\right)$

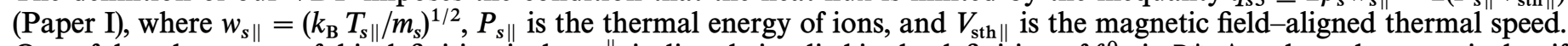
One of the advantages of this definition is that $q_{s 3}^{\|}$is directly implied in the definition of $f_{s}^{0}$ via $D_{s}^{*}$. Another advantage is that if we impose $D_{s}^{*}=0$, then the zeroth-order function $f_{s}^{0}$ is the bi-Maxwellian function used by Demars \& Schunk (1979). The Chew, Goldberger, \& Low (1956) model corresponds to the set of equations obtained with $D_{s}^{*}=0$ and $\phi_{s}=0$. Our model of the VDF is the Grad solution (Grad 1958) when we impose the condition that $T_{s \|}=T_{s \perp}$ and $q_{s 3}^{\|}=0$ in our zeroth-order function $f_{s}^{0}$ and construct the polynomial development following the rules of Mintzer.

\subsection{The 16 Moment Transport Equations}

The method of constructing the transport equations has been explained by Burgers (1969) and Tanenbaum (1967) and used by Demars \& Schunk (1979) for a 16 moment bi-Maxwellian approximation. The notations are defined as follows: $D_{s} / D t=$ $\partial / \partial t+\boldsymbol{u}_{s} \boldsymbol{\nabla}$ is the convective derivative, $e_{s}$ is the charge of species $s, c$ is the speed of light, $\boldsymbol{B}$ is the magnetic field, $\boldsymbol{E}$ is the electric field, $\boldsymbol{G}$ is the acceleration due to gravity, $\partial / \partial t$ is the time derivative, and $\boldsymbol{\nabla}$ is the coordinate space gradient. For the meaning of the mathematical notations see Appendix A. The expressions of the higher order velocity moments as a function of the lower order velocity moments are obtained from the expression of $f_{s G}^{A}$ (see eqs. [1]-[5]). Below, we only present the new truncation assumptions compared to the Demars \& Schunk (1979) model, that is, the expressions of the tensors $\mu_{s \|}$ and $\mu_{s \perp}$ :

$$
\begin{aligned}
\mu_{s \|}=\frac{p_{s \|}}{\rho_{s}}\left[p_{s \perp}\left(\mathbf{I}-e_{3} e_{3}\right)+\tau_{s}-\tau_{s} \cdot e_{3} e_{3}\right. & \left.-e_{3} e_{3} \cdot \tau_{s}\right]+\frac{q_{s 3}^{\perp} q_{s 3}^{\|}}{p_{s \|}}\left(\mathbf{I}-e_{3} e_{3}\right) \\
& +\rho_{s} \frac{\gamma_{s 4}}{p_{s \|}}\left(e_{3} e_{3} \cdot \tau_{s}+\tau_{s} \cdot e_{3} e_{3}\right)+\alpha_{s 2}^{2} \beta_{s 4}\left(q_{s}^{\|} e_{3}+e_{3} q_{s}^{\|}\right)+\left(\rho_{s} \gamma_{s 4}-\alpha_{s 2}^{2} \beta_{s 4} q_{s 3}^{\|}\right) e_{3} e_{3}
\end{aligned}
$$

and

$$
\mu_{s \perp}=\frac{p_{s \perp}}{\rho_{s}}\left[4 p_{s \perp}\left(\mathbf{I}-e_{3} e_{3}\right)+2 p_{s \|} e_{3} e_{3}+6 \tau_{s}-2 \tau_{s} \cdot e_{3} e_{3}-2 e_{3} e_{3} \cdot \tau_{s}\right]+\frac{2 q_{s 3}^{\perp} q_{s 3}^{\|}}{p_{s \|}} e_{3} e_{3},
$$

where

$$
\beta_{s 4}=6 \frac{q_{s 3}^{\|}}{\rho_{s}}\left[\frac{1}{\beta_{s \|}}+2\left(\frac{q_{s 3}^{\|}}{2 \rho_{s}}\right)^{2 / 3}-\beta_{s \|}\left(\frac{q_{s 3}^{\|}}{2 \rho_{s}}\right)^{4 / 3}\right], \quad \gamma_{s 4}=6\left(\frac{q_{s 3}^{\|}}{2 \rho_{s}}\right)^{4 / 3}+\left(\frac{3}{\beta_{s \|}}\right)^{2}-\alpha_{s 2}^{2} \beta_{s 4} \frac{q_{s 3}^{\|}}{\rho_{s}} .
$$

The stress tensor equation is obtained by subtracting $e_{3} e_{3}$ times the parallel energy equation and $\left(\mathrm{I}-\boldsymbol{e}_{3} e_{3}\right)$ times the perpendicular energy equation from the pressure tensor equation, and then the closed system of transport equations is

Continuity:

$$
\frac{\partial}{\partial t}\left(\rho_{s}\right)+\nabla \cdot\left(\rho_{s} u_{s}\right)=0
$$

Momentum:

$$
\rho_{s} \frac{D_{s} \boldsymbol{u}_{s}}{D t}+\nabla_{\perp}\left(p_{s \perp}\right)+\nabla_{\|}\left(p_{s \|}\right)+\nabla \cdot \tau_{s}-\rho_{s} \boldsymbol{G}-n_{s} e_{s}\left(\boldsymbol{E}+\frac{1}{c} \boldsymbol{u}_{s} \times \boldsymbol{B}\right)=\frac{\delta \boldsymbol{M}_{s}}{\delta t}
$$

Parallel energy:

$$
\frac{D_{s} p_{s \|}}{D t}+\nabla \cdot q_{s}^{\|}+p_{s \|}\left(\nabla \cdot u_{s}+2 \nabla_{\|} \cdot \boldsymbol{u}_{s}\right)+2 \tau_{s}: \nabla\left(\boldsymbol{u}_{s \|}\right)=\frac{\delta p_{s \|}}{\delta t}
$$

Perpendicular energy:

$$
\frac{D_{s} p_{s \perp}}{D t}+\nabla \cdot q_{s}^{\perp}+p_{s \perp}\left(\nabla \cdot u_{s}+\nabla_{\perp} \cdot \boldsymbol{u}_{s}\right)+\tau_{s}: \nabla\left(u_{s \perp}\right)=\frac{\delta p_{s \perp}}{\delta t}
$$

Stress tensor:

$$
\begin{aligned}
\frac{D_{\tau_{\tau_{s}}}}{D t} & +\tau_{s}\left(\nabla \cdot \boldsymbol{u}_{s}\right)+\frac{e_{s}}{m_{s} c}\left(B \times \tau_{s}-\tau_{s} \times B\right)+p_{s \|}\left[\nabla_{\|} \boldsymbol{u}_{s}+\left(\nabla_{\|} \boldsymbol{u}_{s}\right)^{\top}-2\left(\nabla_{\|} \cdot \boldsymbol{u}_{s}\right) e_{3} e_{3}\right] \\
& +p_{s \perp}\left[\nabla_{\perp} \boldsymbol{u}_{s}+\left(\nabla_{\perp} \boldsymbol{u}_{s}\right)^{\top}-\left(\nabla_{\perp} \cdot \boldsymbol{u}_{s}\right)\left(\mathbf{I}-e_{3} e_{3}\right)\right]+\tau_{s} \cdot \nabla u_{s}+\left(\tau_{s} \cdot \nabla u_{s}\right)^{\top}-2\left(\tau_{s}: \nabla u_{s \|}\right) e_{3} e_{3}-\left(\tau_{s}: \nabla u_{s \perp}\right)\left(I-e_{3} e_{3}\right) \\
& +\nabla_{\|} q_{s}^{\|}+\left(\nabla_{\|} q_{s}^{\|}\right)^{\top}-2\left(\nabla_{\|} \cdot q_{s}^{\|}\right) e_{3} e_{3}+\frac{1}{2} \nabla_{\perp}\left(q_{s}^{\perp}+q_{s \|}^{\perp}\right)+\frac{1}{2}\left[\nabla_{\perp}\left(q_{s}^{\perp}+q_{s \|}^{\perp}\right)\right]^{\top}-\frac{1}{2}\left(\nabla_{\perp} \cdot q_{s}^{\perp}\right)\left(I-e_{3} e_{3}\right)=\frac{\delta \tau_{s}}{\delta t},
\end{aligned}
$$


Parallel heat flux:

$$
\begin{aligned}
\frac{D_{s} q_{s}^{\|}}{D t} & +\boldsymbol{q}_{s}^{\|} \cdot \boldsymbol{\nabla} \boldsymbol{u}_{s}+\boldsymbol{q}_{s}^{\|}\left(\boldsymbol{\nabla} \cdot \boldsymbol{u}_{s}\right)-\frac{e_{s}}{m_{s} c}\left(\boldsymbol{q}_{s}^{\|} \times \boldsymbol{B}\right)+\frac{k_{\mathrm{B}}}{m_{s}} \nabla_{\perp}\left(T_{s \|} p_{s \perp}\right)+\frac{k_{\mathrm{B}}}{m_{s}}\left[\boldsymbol{\nabla} \cdot\left(T_{s \|} \tau_{s}\right) \cdot\left(\mathbf{I}-\boldsymbol{e}_{3} \boldsymbol{e}_{3}\right)-\nabla_{\|}\left(T_{s \|} \tau_{s}\right)\right] \\
& +\nabla_{\perp}\left(\frac{q_{s 3}^{\perp} q_{s 3}^{\|}}{p_{s \|}}\right)+\nabla \cdot\left(\frac{\gamma_{s 4} \tau_{s}}{p_{s \|}}\right) \cdot \boldsymbol{e}_{3} \boldsymbol{e}_{3}+\nabla_{\|} \cdot\left(\frac{\gamma_{s 4} \tau_{s}}{p_{s \|}}\right)+\left[\nabla \cdot\left(\alpha_{s 2}^{2} \beta_{s 4} \boldsymbol{q}_{s}^{\|}\right)\right] e_{3}+\boldsymbol{e}_{3}^{\mathrm{\top}} \cdot\left[\nabla\left(\alpha_{s 2}^{2} \beta_{s 4} \boldsymbol{q}_{s}^{\|}\right)\right] \\
& -2 \nabla_{\|}\left(\alpha_{s 2}^{2} \beta_{s 4} q_{s 3}^{\|}\right)+\nabla_{\|}\left\{\rho_{s}\left[6\left(\frac{q_{s 3}^{\|}}{2 \rho_{s}}\right)^{4 / 3}+\frac{3}{\beta_{s \|}^{2}}\right]\right\} \\
& +\left[\frac{D_{s} \boldsymbol{u}_{s}}{D t}-\boldsymbol{G}-\frac{e_{s}}{m_{s}}\left(\boldsymbol{E}+\frac{1}{c} \boldsymbol{u}_{s} \times \boldsymbol{B}\right)\right] \cdot\left[p_{s \|} \mathbf{I}+2 \boldsymbol{e}_{3} \boldsymbol{e}_{3} \cdot\left(\tau_{s}+p_{s \|} \mathbf{I}\right)\right] \\
& +2\left[\boldsymbol{q}_{s}^{\|} \cdot \nabla_{\perp} \boldsymbol{u}_{s \|}+\boldsymbol{q}_{s}^{\|}\left(\nabla_{\|} \cdot \boldsymbol{u}_{s}\right)+\left(\nabla_{\perp} \boldsymbol{u}_{s \|}\right) \cdot \boldsymbol{q}_{s}^{\perp}\right]=\frac{\delta \boldsymbol{q}_{s}^{\|}}{\delta t},
\end{aligned}
$$

Perpendicular heat flux:

$$
\begin{aligned}
\frac{D_{s} \boldsymbol{q}_{s}^{\perp}}{D t} & +\boldsymbol{q}_{s}^{\perp} \cdot \nabla \boldsymbol{u}_{s}+\boldsymbol{q}_{s}^{\perp}\left(\nabla \cdot \boldsymbol{u}_{s}\right)-\frac{e_{s}}{m_{s} c}\left(\boldsymbol{q}_{s}^{\perp} \times \boldsymbol{B}\right)+\frac{k_{\mathrm{B}}}{m_{s}}\left[2 \nabla_{\perp}\left(T_{s \perp} p_{s \perp}\right)+\nabla_{\|}\left(T_{s \perp} p_{s \|}\right)\right. \\
& \left.-\nabla_{\|} \cdot\left(T_{s \perp} \tau_{s}\right)+\nabla \cdot\left(T_{s \perp} \tau_{s}\right) \cdot\left(3 \mathbf{I}-e_{3} e_{3}\right)\right]+\nabla_{\|}\left(\frac{q_{s 3}^{\perp} q_{s 3}^{\|}}{p_{s \|}}\right)+\frac{1}{2} \boldsymbol{q}_{s}^{\perp} \cdot \nabla_{\perp} \boldsymbol{u}_{s \perp} \\
& +\frac{1}{2}\left[\left(\nabla_{\perp} \boldsymbol{u}_{s \perp}\right) \cdot \boldsymbol{q}_{s}^{\perp}+\left(\nabla_{\perp} \cdot \boldsymbol{u}_{s}\right)\left(\mathbf{I}+\boldsymbol{e}_{3} \boldsymbol{e}_{3}\right) \cdot \boldsymbol{q}_{s}^{\perp}\right]+\boldsymbol{q}_{s}^{\perp} \cdot \nabla_{\|} \boldsymbol{u}_{s \perp}+\left(\nabla_{\|} \boldsymbol{u}_{s \perp}\right) \cdot \boldsymbol{q}_{s}^{\|} \\
& +\left[\frac{D_{s} \boldsymbol{u}_{s}}{D t}-\boldsymbol{G}-\frac{e_{s}}{m_{s}}\left(\boldsymbol{E}+\frac{1}{c} \boldsymbol{u}_{s} \times \boldsymbol{B}\right)\right] \cdot\left[p_{s \perp} \mathbf{I}+\left(\mathbf{I}-\boldsymbol{e}_{3} \boldsymbol{e}_{3}\right) \cdot\left(\tau_{s}+p_{s \perp} \mathbf{I}\right)\right]=\frac{\delta \boldsymbol{q}_{s}^{\perp}}{\delta t} .
\end{aligned}
$$

We verify the results presented in this subsection in comparison to those presented by Demars \& Schunk (1979) by setting $D_{s}^{*}$ equal to zero, as in the previous subsection. The new terms that correspond to the second, third, and fourth lines of equation (14) and the third term of the second line in equation (15) change into gradients involving only the stress tensor and the parallel energy. The heat-flux gradients and the nonlinear heat-flux terms in equations (14) and (15) are then new terms, differing from previous moment approach models. The right-hand side of each equation (see eqs. [9]-[15]) is the velocity moment of Boltzmann's collision integral. These quantities will be evaluated in a forthcoming paper for typical interparticle force and particle populations.

We have made the same development for a 9 moment approximation, for which we used the macroscopic parameters $n_{s}, \boldsymbol{u}_{s}$, $T_{s \|}, T_{s \perp}$, and $\boldsymbol{q}_{s}$ (see Appendix B) as independent variables. The advantage of such a model is its capacity to provide large temperature anisotropy and large heat-flux values in a simple way. But, obviously, the stress terms are not taken into account, and in a 16 moment approximation neither is the heat-flux anisotropy. The definition of $f_{s G}^{0}$ is very close to the definition given for the 16 moment approximation, the only difference being the relation

$$
\int_{V} c_{s}^{2} c_{s 3} f_{s G}^{0}\left(r, c_{s}, t\right) d c_{s}=\int_{V} c_{s}^{2} c_{s 3} f_{s}^{E}\left(r, c_{s}, t\right) d c_{s}
$$

instead of an equivalent relation, where $c_{s}^{2}$ is replaced by $c_{s \|}^{2}$ for a 16 moment approximation. The exact solution is $f_{s}^{E}$. The equations for the 9 moment approximation (see eqs. [9] and [B5]-[B8]), the definition of the derived polynomial expansion (see eq. [B1]), and the expressions of the higher order velocity moments as a function of the lower order moments (see eqs. [B2]-[B4]) are given in Appendix B.

\section{PROPERTIES OF THE GENERALIZED TRANSPORT EQUATIONS}

\subsection{The Hyperbolicity Criterion}

The hyperbolicity criterion is a necessary condition for a linearized system of transport equations to be well posed, being the condition for the existence of a stable and unique solution of a linearized problem associated with Cauchy boundary conditions. Cordier (1994a, 1994b) and Cordier \& Girard (1996) have described a mathematical method for studying the hyperbolicity of a system of transport equations. These works have been applied to systems derived from a polynomial expansion function based on a bi-Maxwellian function for 8, 10,13, and 16 independent moments. In this section we discuss the hyperbolicity criterion relative to the set of transport equations (9)-(15).

The first step is to project equations (9)-(15) onto the direction of the magnetic field. This amounts to supposing that the motion of the particles is confined along the magnetic field lines, which allows us to reduce the number of independent moments to consider ( 6 in the case of a 16 moment approximation). We note that this assumption of gyrotropic-dominated plasmas is often made in studies of the polar and solar winds (Palmadesso, Ganguli, \& Mitchell 1988; Demars \& Schunk 1991). If we write the velocity $v$ in the form $\left(\boldsymbol{v}_{\|}, v_{\perp}\right)$ where $v_{\|}$and $v_{\perp}$ are, respectively, the components of $v$ parallel and 
perpendicular to the magnetic field, we then have $\boldsymbol{u}_{s \perp}=\left\langle\boldsymbol{v}_{s \perp}\right\rangle=0$, and the state variables depend only on $z: n_{s}(z, t), u_{s 3}(z, t)$, $p_{s \|}(z, t), p_{s \perp}(z, t), q_{s 3}^{\|}(z, t)$, and $q_{s 3}^{\perp}(z, t)$. Furthermore, the pressure tensor can be written in the form

$$
\mathbf{P}=\left(\begin{array}{ccc}
p_{s \perp} & 0 & 0 \\
0 & p_{s \perp} & 0 \\
0 & 0 & p_{s \|}
\end{array}\right)
$$

which implies that the stress tensor $\tau_{s}=0$.

We also neglect the collisional terms, and consequently the dissipative effects that would have generated a less restrictive limit. But this corresponds to the situation observed in the solar wind for large heliocentric distances. The one-dimensional system projected along the magnetic field is given by

$$
\begin{gathered}
\frac{\partial n_{s}}{\partial t}+u_{s 3} \frac{\partial n_{s}}{\partial z}+n_{s} \frac{\partial u_{s 3}}{\partial z}=0 \\
\frac{\partial u_{s 3}}{\partial t}+u_{s 3} \frac{\partial u_{s 3}}{\partial z}+\frac{k_{\mathrm{B}} T_{s \|}}{\rho_{s}} \frac{\partial n_{s}}{\partial z}+\frac{k_{\mathrm{B}}}{m_{s}} \frac{\partial T_{s \|}}{\partial z}=0 \\
\frac{\partial T_{s \|}}{\partial t}+u_{s 3} \frac{\partial T_{s \|}}{\partial z}+2 T_{s \|} \frac{\partial u_{s 3}}{\partial z}+\frac{1}{n_{s} k_{\mathrm{B}}} \frac{\partial q_{s 3}^{\|}}{\partial z}=0, \\
\frac{\partial T_{s \perp}}{\partial t}+u_{s 3} \frac{\partial T_{s \perp}}{\partial z}+\frac{1}{n_{s} k_{\mathrm{B}}} \frac{\partial q_{s 3}^{\perp}}{\partial z}=0, \\
\frac{\partial q_{s 3}^{\|}}{\partial t}-\frac{m_{s}}{2^{1 / 3}}\left(\frac{q_{s 3}^{\|}}{\rho_{s}}\right)^{4 / 3} \frac{\partial n_{s}}{\partial z}+4 q_{s 3}^{\|} \frac{\partial u_{s 3}}{\partial z}+\frac{3 n_{s} k_{\mathrm{B}}^{2} T_{s \|}}{m_{s}} \frac{\partial T_{s \|}}{\partial z}+\left[u_{s 3}+2^{5 / 3}\left(\frac{q_{s 3}^{\|}}{\rho_{s}}\right)^{1 / 3}\right] \frac{\partial q_{s 3}^{\|}}{\partial z}=0,
\end{gathered}
$$

and

$$
\frac{\partial q_{s 3}^{\perp}}{\partial t}-\frac{q_{s 3}^{\|} q_{s 3}^{\perp}}{n_{s}^{2} k_{\mathrm{B}} T_{s \|}} \frac{\partial n_{s}}{\partial z}+2 q_{s 3}^{\perp} \frac{\partial u_{s 3}}{\partial z}-\frac{q_{s 3}^{\|} q_{s 3}^{\perp}}{n_{s} k_{\mathrm{B}} T_{s \|}^{2}} \frac{\partial T_{s \|}}{\partial z}+\frac{n_{s} k_{\mathrm{B}}^{2} T_{s \|}}{m_{s}} \frac{\partial T_{s \perp}}{\partial z}+\frac{q_{s 3}^{\perp}}{m_{s} k_{\mathrm{B}} T_{s \|}} \frac{\partial q_{s 3}^{\|}}{\partial z}+\left(u_{s 3}+\frac{q_{s 3}^{\|}}{n_{s} k_{\mathrm{B}} T_{s \|}}\right) \frac{\partial q_{s 3}^{\perp}}{\partial z}=0 .
$$

As explained by Cordier (1994a) in a simple species case, the one-dimensional system (see eqs. [16]-[21]) has a schematic form of

$$
\frac{\partial U}{\partial t}+\mathbf{A}(U) \frac{\partial U}{\partial z}=0
$$

where $U$ is the vector $\left(n_{s}, u_{s 3}, p_{s \|}, p_{s \perp}, q_{s 3}^{\|}, q_{s 3}^{\perp}\right)^{\top}$ and $\mathbf{A}(U)$ is a $6 \times 6$ matrix.

In the case of a perturbation of the form $U=U_{0}+U_{1} \exp [i(k z-\omega t)]$, the solution of the characteristic polynomial of $\mathbf{A}(U)$ is the phase velocities $\omega / k$ of the ion waves generated by this perturbation. The condition for the system to be hyperbolic is that the phase velocities all be real. In order to determine these phase velocities, we calculate the characteristic polynomial of $\mathbf{A}(U)$, which has the form

$$
P(\lambda)=\left(X^{2}+\frac{1}{4} \epsilon^{3} X-1\right)\left[X^{4}+2 \epsilon X^{3}-6 X^{2}+\epsilon\left(\epsilon^{2}-6\right) X+3+\frac{1}{8} \epsilon^{4}\right],
$$

where $X=\left(u_{s 3}-\lambda\right) / w_{s \|}, \epsilon=\left(4 q_{s 3}^{\|} / \rho_{s} w_{s \|}^{3}\right)^{1 / 3}$, and $\lambda$ is a characteristic velocity of $\mathbf{A}(U)$. The parameter $w_{s \|}$ is defined as $w_{s \|}=\left(k_{\mathrm{B}} T_{s \|} / m_{s}\right)^{1 / 2}$. The first bracket always has two real roots. But the second part of $P$ has four real roots if and only if $|\epsilon|<2.00$. The condition for hyperbolicity can also be written in the form

$$
\left|q_{s 3}^{\|}\right|<2.00 \rho_{s} w_{s \|}^{3} .
$$

This study is similar to the work of Palmadesso et al. (1988), who solved the dispersion relation issuing from a biMaxwellian 16 moment approximation for large-scale magnetospheric ionospheric dynamics and obtained unstable wave modes for certain values of the macroscopic parameters; specifically, for heat-flux values above $0.88 \rho_{s} w_{s \|}^{3}$. In fact, a study of the solutions of the dispersion relation allows us to better illustrate the consequences of the nonhyperbolicity of a moment approach. The first bracket of the characteristic polynomial (see eq. [23]) always has two roots:

$$
\lambda=u_{s 3}+\frac{w_{s \|}}{2}\left\{\left(\frac{\epsilon}{2}\right)^{2} \pm\left[\left(\frac{\epsilon}{2}\right)^{4}+4\right]^{1 / 2}\right\}
$$

Palmadesso et al. (1988) explain that these phase velocities are associated with perpendicular temperature and heat-flux waves. The second bracket has four real or complex roots, which are represented in Figure 1 as a function of the $\epsilon$ parameter. The two phase velocities that are nearly independent of $\epsilon$ are parallel acoustic waves, and the two others are thermal waves, according to the very similar work of Palmadesso et al. (1988). When the $\epsilon$ parameter is equal to \pm 2 , two waves couple and generate an unstable wave above this value in $q_{s 3}^{\|}$. Palmadesso et al. (1988) emphasize that such an unstable wave is the consequence of the truncation of the moment approach. Indeed, with a moment approach, a perturbation of the higher 


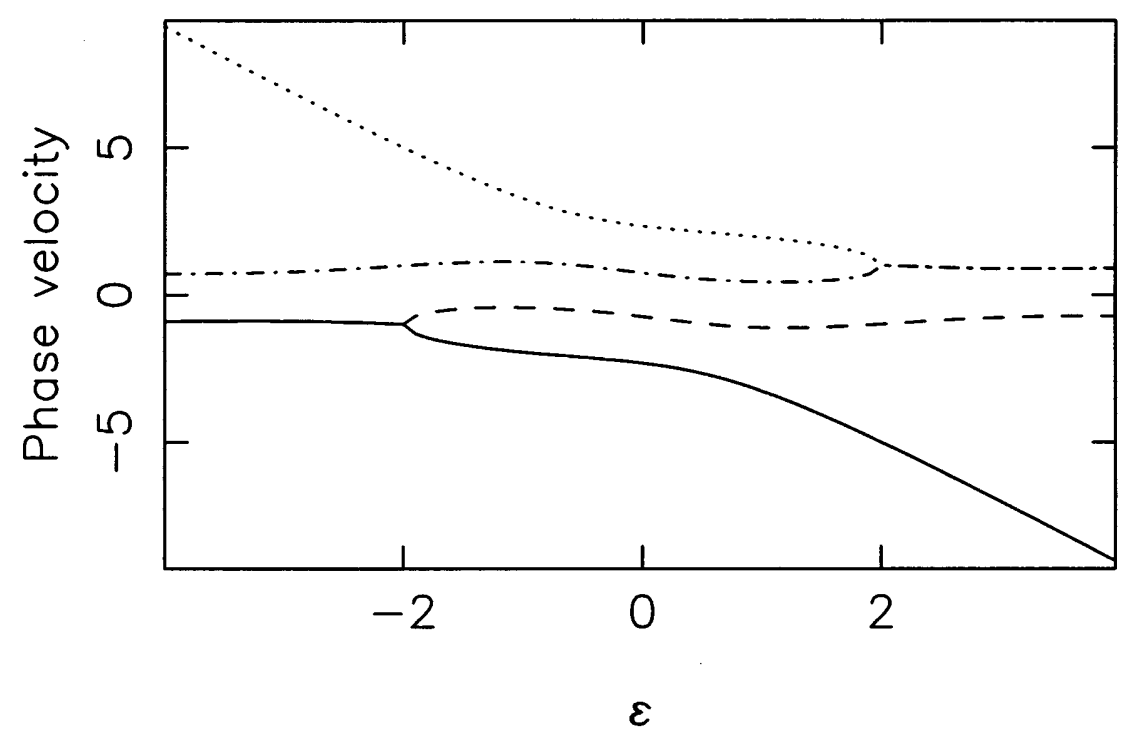

FIG. 1.-Dependence of the four phase velocities solution of the characteristic polynomial (see eq. [23]) as a function of the $\epsilon$ parameter. From $|\epsilon|>2$., we have represented the real part of the complex solution.

moment of the model cannot be dissipated in the fine structure, as with a kinetic model, because the highest order moments, which are not taken into account as a result of the truncation, play the role of the fine structure in a moment approach. Therefore, the highest moment is perturbed by an artificial, undamped wave that can then interact with another real wave, as shown in Figure 1, and generate an unstable wave. Equation (24) must be understood as a necessary condition for the stability of the solution in the case of a linearized problem. It is a rough estimate of the highest value of the heat flux that can be modeled with the generalized polynomial approach in applications to noncollisional astrophysical winds.

\subsection{The Moment Realizability}

In recent papers, several authors (Levermore et al. 1995; Levermore 1995; Groth et al. 1996) have developed new methods for estimating the domain of validity of the moment closure associated with the polynomial expansion function. These authors have noted that the first-order Chapman-Enskog (Chapman \& Cowling 1970) model, which leads to Navier-Stokes equations, always has negative values when the Navier-Stokes equations have proved to be valid for flows in the continuum regime. Thus, it seems that the positivity of the polynomial expansion function that generates the set of transport equations is an overly restrictive condition. A more realistic criterion is to find the conditions that will ensure the existence of a positive distribution function that could generate the same set of velocity moments.

According to Hamburger (1944), a necessary condition for a set of moments of a function to generate a positive function is that the matrix $\mathbf{M}$, defined as $\mathbf{M}=\left\langle U U^{\top} F_{s}^{N}\right\rangle$, must be positive definite. $U=\left(1, c_{s i}, c_{s i} c_{s j}, \ldots\right)^{\mathrm{T}}$ corresponds to the velocitymoment space, and $F_{s}^{N}$ is any VDF whose velocity moments are used in the approximation considered. Obviously, the derived criterion cannot be used in practice. In order to construct a useful mathematical condition, Levermore et al. have considered the subspace generated by the velocity moments corresponding to the usual hydrodynamic approximations. Indeed, according to Levermore (1995), every closure assumption of a polynomial expansion method must recover this level of description. Thus, we determine the condition of realizability for $U=\left(1, c_{s i}, c_{s}^{2}\right)^{\top}$ that is the velocity moment subspace used in a hydrodynamic approximation. This condition is obviously a restriction of the exact criterion that corresponds to the 16 moment approximation. In fact, the exact condition for the positivity and definition of the matrix $\mathbf{M}$ should have been calculated for $U=\left(1, c_{s i}, c_{s i} c_{s j}, c_{s \perp}^{2} c_{s i}, c_{s \|}^{2} c_{s i}\right)^{\top}$ (Reed \& Simon 1975). But in such a case, M would be much too large (with $16 \times 16$ elements) to give a useful criterion. So we restrain our analysis, following the advice of Levermore (1995).

The matrix corresponding to a 16 moment approximation is then

$$
\mathbf{M}_{16}=\left(\begin{array}{ccccc}
\rho_{s} & 0 & 0 & 0 & 2 p_{s \perp}+p_{s \|} \\
0 & p_{s 11} & p_{s 12} & p_{s 13} & 2 q_{s 1} \\
0 & p_{s 21} & p_{s 22} & p_{s 23} & 2 q_{s 2} \\
0 & p_{s 31} & p_{s 32} & p_{s 33} & 2 q_{s 3} \\
2 p_{s \perp}+p_{s \|} & 2 q_{s 1} & 2 q_{s 2} & 2 q_{s 3} & r_{s}
\end{array}\right),
$$

where

$$
r_{s}=\frac{8 p_{s \perp}^{2}}{\rho_{s}}+\frac{4 p_{s \perp} p_{s \|}}{\rho_{s}}+\frac{3 p_{s \|}^{2}}{\rho_{s}}+6 \rho_{s}\left(\frac{q_{s 3}^{\|}}{\rho_{s}}\right)^{4 / 3}
$$

For a symmetric and diagonalizable matrix such as $\mathbf{M}_{16}$, a condition equivalent to its positivity is that all the determinants of the submatrices must be positive. But for such an approximation, the moment realizability is not easy to evaluate. In 
one-dimensional flows $\left(q_{s 1}=q_{s 2}=p_{s 12}=p_{s 13}=p_{s 23}=0, p_{s 33}=p_{s \|}\right.$, and $\left.p_{s 11}=p_{s 22}=p_{s \perp}\right)$, the matrix $\mathbf{M}_{16}$ (see eq. [25]) is easier to analyze. We find as a condition for the moment realizability in one-dimensional flows

$$
\rho_{s}>0, \quad p_{s \perp}>0, \quad p_{s \|}>0, \quad \text { and } \quad 2 p_{s \perp}^{2}+p_{s \|}^{2}+3 \rho_{s}^{2}\left(\frac{q_{s 3}^{\|}}{\rho_{s}}\right)^{4 / 3}-2 \rho_{s} \frac{q_{s 3}^{2}}{p_{s \|}}>0 .
$$

This condition is a more general criterion than the hyperbolicity condition. In this case, the limitation on the normalized heat flux depends on both temperature and heat-flux anisotropies. It is significant if we compare it to the equivalent conditions determined for a bi-Maxwellian model, which we present in the following section.

\section{DISCUSSION}

Only the equations of the heat-flux vectors (eqs. [14] and [15]) are different from those in the model of Demars \& Schunk (1979). In our model, $\boldsymbol{\mu}_{s}^{\|}$and $\boldsymbol{\mu}_{s}^{\perp}$ depend on the heat fluxes $\boldsymbol{q}_{s}^{\|}$and $q_{s 3}^{\perp}$. This property is a direct consequence of the zeroth-order nonequilibrium distribution function bringing a heat flux, whereas the bi-Maxwellian approximation of $\mu_{s}^{\|}$and $\mu_{s}^{\perp}$ does not take into account the asymmetric character of the microscopic nonequilibrium state. The new expression of the velocity moment $\left\langle c_{s \|}^{4}\right\rangle$ is $\left\langle c_{s \|}^{4}\right\rangle_{\text {Max }}+\left(q_{s 3}^{\|} / \rho_{s}\right)^{4 / 3}$. In this expression, the new term $\left(\boldsymbol{q}_{s 3}^{\|} / \rho_{s}\right)^{4 / 3}$ is derived from the suprathermal long tail of the microscopic state.

The consequence of this new truncation of the generalized approach is to modify the equations of the heat-flux vector related to the direction of the magnetic field by the introduction of heat-flux gradients and nonlinear terms. The onedimensional system of transport equations is a good illustration of this fact. In contrast to the equivalent equation obtained by Demars \& Schunk (1979), in the equation for $q_{s 3}^{\perp}$ (see eq. [21]), the variations of $n_{s}, q_{s 3}^{\|}$, and $T_{s \|}$ as a function of the position $z$ along the magnetic field are taken into account. In the equation for $q_{s 3}^{\|}$, the spatial gradient of $n_{s}$ in the direction $z$ of the magnetic field does not appear in the equivalent equation of the bi-Maxwellian approach (Demars \& Schunk 1979), and the multiplier to the spatial gradient of $q_{s 3}^{\|}$is modified. In consequence, the equations of the heat-flux vector in the direction of the magnetic field are more strongly coupled to the other equations than with a bi-Maxwellian approximation. The Monte Carlo simulations (Barghouthy, Barakat, \& Schunk 1993) show correlated temperature and heat-flux profiles with larger temperature and heat-flux anisotropies than with a bi-Maxwellian model. Moreover, they show large gradients of the heat flux in the transitional regions, which are then better accounted for thanks to the heat-flux gradients of equations (20) and (21). On the other hand, a good estimate of the pressure anisotropy, which depends on a good estimate of the heat fluxes, is important to accurately reproduce the important effects of the mirror force(Spitzer 1952).

We are able to evaluate the greater accuracy of the truncation of the system using the realizability method that we present in $\S 3.2$. If we apply this analysis to the bi-Maxwellian model for one species and for one-dimensional flows, we find the following conditions:

$$
\rho_{s}>0, \quad p_{s \perp}>0, \quad p_{s \|}>0, \quad \text { and } \quad 2 p_{s \perp}^{2}+p_{s \|}^{2}-2 \rho_{s} \frac{q_{s 3}^{2}}{p_{s \|}}>0 .
$$

If we compare equation (27) to the conditions obtained for a similar application defined in equation (26), we can establish that the moment realizability provides a more restrictive condition on the velocity moments for a bi-Maxwellian polynomial expansion than for a generalized model. This is a consequence of the asymmetric character of the observed VDF, which is better taken into account with the new term $\left(q_{s 3}^{\|} / \rho_{s}\right)^{4 / 3}$ in equation (26).

In order to justify the interest of a greater coupling between the transport equations, we use the hyperbolicity condition as applied by Cordier (1994a, 1994b) to a 16 moment bi-Maxwellian model. The mathematical description of this method is presented in $\S 3.1$. For the same assumptions as used previously, Cordier found the condition

$$
\left|q_{s 3}^{\|}\right|<0.91 \rho_{s} w_{s \|}^{3} .
$$

Once again, we note that the generalized model generates a less restrictive condition (see eq. [24]). Indeed, two phase velocities are associated with an unstable wave in $q_{s 3}^{\|}$when $\left|q_{s 3}^{\|}\right|>2.00 \rho_{s} w_{s \|}^{3}$ for a generalized model. As shown by Palmadesso et al. (1988), this limit derives from the same criterion as those for a 16 moment bi-Maxwellian model.

These necessary conditions (see eqs. [24], [26], [28], and [27]) consist of a limitation of the normalized heat flux by the free-streaming flux, the parallel energy brought by particles whose velocity is the thermal velocity. We propose that these moment equations can accurately describe the nonequilibrium states that may correspond to high values of the heat flux, and could therefore describe the particle nonequilibrium states from collisional to collisionless regions. Indeed, the Navier-Stokes equations, which are well adapted to the description of the collisional regions, are included in the generalized model.

The evolution of the normalized heat flux in the solar wind from 0.3 to $1 \mathrm{AU}$ measured by the Helios solar probe (Marsch et al. 1982) is shown in Figure 2. This figure displays the decrease of the normalized heat flux with heliocentric distance. This decrease could be explained by wave-particle interactions. Figure 2 could also explain the difficulties of Demars $\&$ Schunk (1991), who used a 16 moment bi-Maxwellian model to simulate the solar wind. In that paper, they show that a singular point in the parallel heat flux radial evolution appears around 0.4 AU. In Figure 2, the value of the normalized heat flux largely exceeds the limit defined by equations (27) and (28) (taking into account measurement errors in the normalized heat flux; Marsch et al. 1982).

The effect of the thermal force, a force that depends on the heat flux and appears in the velocity equation, needs to be accurately determined. According to Dorelli \& Scudder (1996), the Spitzer law is not valid in the solar corona, and according to Demars \& Schunk (1991), neither is the law deduced from the 16 moment bi-Maxwellian model. In the generalized model, 


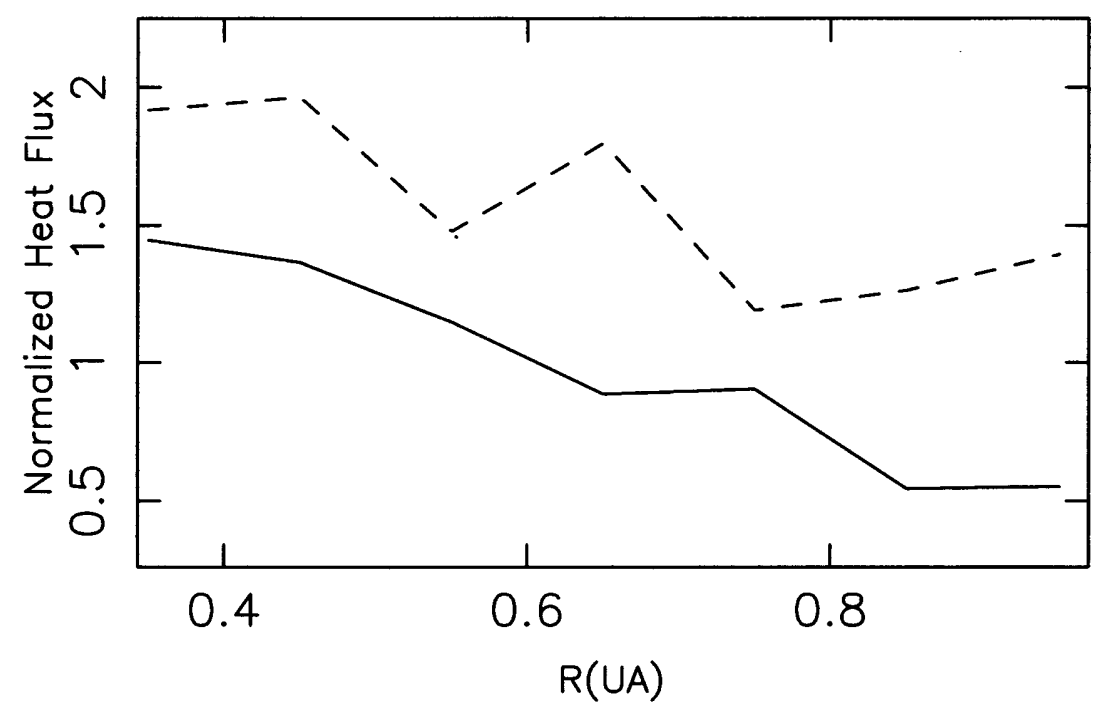

FIG. 2.-Normalized heat flux from 0.3 to $1 \mathrm{AU}$, obtained from the values of the macroscopic parameters determined by Marsch (1982) and Marsch et al. (1982) from the Helios measurements. The solid line corresponds to the slow winds $\left(300-400 \mathrm{~km} \mathrm{~s}^{-1}\right)$ and the dashed line to the fast winds $\left(600-700 \mathrm{~km} \mathrm{~s}^{-1}\right)$.

the thermal force depends on more macroscopic parameter evolutions. This model should then be better adapted to model and so determine the right effects of the thermal force in the acceleration processes, and so to explain why in Figure 2 the normalized heat flux is greater for fast winds than for slow winds.

In the polar wind, simulations predict that for all species the heat flux greatly exceeds the limiting value that guarantees the hyperbolicity of the system of transport equations associated with a 16 moment bi-Maxwellian approximation (Robineau, Blelly, \& Fontanari 1996). Although estimated in the collisionless limit, these results show, as for the solar wind, the difficulties in modeling states far from local equilibrium with a bi-Maxwellian approximation.

\section{CONCLUSION}

In Paper I, we analyzed the microscopic properties of a generalized moment approach to stellar atmosphere expansions, in particular to the solar and polar winds, the properties of which are now better known thanks to several probes (Helios 1 and 2, Interplanetary Monitoring Platform (IMP) 8, Ulysses, and $\mathrm{SOHO}$ ) or by Monte Carlo simulations. This second paper provides the associated set of generalized transport equations, constructed using the method of Burgers (1969) and Tanenbaum (1967) and previously used by Demars \& Schunk (1979). We have not given the corresponding collisional terms. Two levels of approximation are considered. The 16 moment approximation can describe temperature and heat-flux anisotropies using the same independent macroscopic parameters as the 16 moment bi-Maxwellian approximation of Demars \& Schunk (1979); the 9 moment approximation is a simpler model, without pressure-tensor or heat-flux anisotropy. We also give the higher order velocity moment closure assumptions for both approximations. Well adapted to describing thermalized state and temperature anisotropy, a 16 moment bi-Maxwellian approximation is not able to model large heat-flux values. However, the heat-flux radial dependence in the solar wind shows the importance of this parameter in describing the internal energetic state of the protons. In order to be able to accurately reproduce the heat-flux evolution as measured by probes in the solar and polar winds from collisional to collisionless regions, we have improved the moment approach.

A comparison with the bi-Maxwellian model is provided in order to illustrate this improvement. To this end, we use two different analyses of the system of equations: the hyperbolicity approach, which studies the well-posed nature of the set of transport equations, and a realizability analysis, which evaluates the physical nature of the moment closure. Both analyses show that the description of the heat flux in the transitional collisional region is improved.

Although it is mainly applied to the solar wind, the generalized moment approach can also model stellar atmosphere and planetary wind expansions.

We thank S. Cordier, P.-L. Blelly, and A. Mangeney for helpful comments on this paper.

\section{APPENDIX A}

\section{MATHEMATICAL OPERATIONS}

$\mathbf{A}$ and $\mathbf{B}$ are tensors of dimension 2. The contracted product of two tensors is defined as

$$
(\mathbf{A} \cdot \mathbf{B})_{i j}=A_{i k} B_{k j}
$$


with the summation convention for equal component indices. The double contracted product of two tensors is defined as

$$
\mathbf{A}: \mathbf{B}=A_{i k} B_{k i} .
$$

We also define the products between a tensor $\mathbf{A}$ and a vector $\boldsymbol{u}$ by

$$
\begin{gathered}
(\boldsymbol{u} \cdot \mathbf{A})_{i}=u_{k} A_{k i}, \\
(\mathbf{A} \times \boldsymbol{u})_{i j}=\epsilon_{i l k} A_{l j} u_{k},
\end{gathered}
$$

where $\epsilon_{i l k}$ is equal to -1 or 1 when ilk is an even or odd permutation of $1,2,3$, respectively, and equal to zero if two indices are equal. With two vectors $u$ and $v$, this product is defined as usual by

$$
(u \times v)_{k}=\epsilon_{i j k} u_{i} v_{j} .
$$

The operations with a gradient $\nabla$ are defined as

$$
\begin{gathered}
(\boldsymbol{\nabla} \cdot \mathbf{A})_{k}=\frac{\partial A_{k l}}{\partial x_{l}}, \\
(\nabla \boldsymbol{u})_{i j}=\frac{\partial u_{j}}{\partial x_{i}} .
\end{gathered}
$$

We have defined two operations with subscripts $\perp$ and $\|$ as

$$
\nabla_{\perp} \boldsymbol{u}=\left(\begin{array}{ccc}
u_{1} / \partial x_{1} & \partial u_{2} / \partial x_{1} & \partial u_{3} / \partial x_{1} \\
\partial u_{1} / \partial x_{2} & \partial u_{2} / \partial x_{2} & \partial u_{3} / \partial x_{2} \\
0 & 0 & 0
\end{array}\right),
$$

\section{APPENDIX B}

\section{THE 9 MOMENT TRANSPORT EQUATIONS}

The velocity moments used in a 9 moment approximation are the density $n_{s}$, and

$\boldsymbol{u}_{s}=\left\langle\boldsymbol{v}_{s}\right\rangle$

Species drift velocity

$T_{s \|}=\left(m_{\mathrm{s}} / k_{\mathrm{B}}\right)\left\langle c_{s \|}^{2}\right\rangle \quad$ Parallel temperature

$T_{s \perp}=\left(m_{s} / 2 k_{\mathrm{B}}\right)\left\langle c_{s \perp}^{2}\right\rangle \quad$ Perpendicular temperature

$\boldsymbol{q}_{s}=\frac{1}{2} \rho_{s}\left\langle c_{s}^{2} \boldsymbol{c}_{s}\right\rangle \quad$ Heat-flux vector for energy.

The term $f_{s G}^{0}$ is the same as for a 16 moment approximation, but with

$$
D_{s}^{*}=\left(\frac{q_{s 3}}{\rho_{s}}\right)^{1 / 3} .
$$

The terms $E^{*}$ and $T_{0}$ are defined as for a 16 moment approach. The polynomial part of the velocity distribution function is given by

$$
\phi_{s}=2\left[c_{s}^{2}-\left(\frac{4}{\beta_{s \perp}}+\frac{1}{\beta_{s \|}}\right)\right] \frac{\boldsymbol{q}_{s} \cdot c_{s \perp}}{\rho_{s} \alpha_{s 1}^{\prime \prime}},
$$

where $\beta_{s \|}$ and $\beta_{s \perp}$ are defined in the same way as for the 16 moment approximation (see eq. [4]) and

$$
\alpha_{s 1}^{\prime \prime}=\frac{8}{\beta_{s \perp}^{3}}+\frac{2}{\beta_{s \perp} \beta_{s \|}^{2}}+\frac{6}{\beta_{s \perp}}\left(\frac{q_{s 3}}{\rho_{s}}\right)^{4 / 3} .
$$

In order to close the left-hand side of the transport equations, we need the expression of the velocity moments $\mathbf{P}_{s}, \mathbf{Q}_{s}$, and $\boldsymbol{\mu}_{s}=\frac{1}{2} \rho_{s}\left\langle c_{s}^{2} c_{s} \boldsymbol{c}_{s}\right\rangle$. With the new expression of the approximation of the velocity distribution function, we obtain

$$
\mathbf{P}_{s}=\frac{\rho_{s}}{\beta_{s \perp}} \mathbf{I}+\left(\frac{\rho_{s}}{\beta_{s \|}}-\frac{\rho_{s}}{\beta_{s \perp}}\right) e_{3} e_{3}+\frac{4 q_{s 3}}{\rho_{s} \beta_{s \perp} \alpha_{s 1}^{\prime \prime}}\left(q_{s} e_{3}+e_{3} q_{s}-2 q_{s 3} e_{3} e_{3}\right),
$$




$$
\begin{gathered}
\mathbf{Q}_{s}=\frac{4}{\beta_{s \perp}^{3} \alpha_{s 1}^{\prime \prime}}\left(\mathbf{I} q_{s}+q_{s} \mathbf{I}+e_{1} q_{s} e_{1}+e_{2} q_{s} e_{2}+e_{3} q_{s} e_{3}\right)-\frac{4}{\beta_{s \perp}^{3} \alpha_{s 1}^{\prime \prime}} q_{s 3}\left(I e_{3}+e_{3} \mathbf{I}+e_{1} e_{3} e_{1}+e_{2} e_{3} e_{2}+e_{3} e_{3} e_{3}\right) \\
+2\left(1-\frac{10}{\beta_{s \perp}^{3} \alpha_{s 1}^{\prime \prime}}\right)\left(e_{3} e_{3} q_{s}+q_{s} e_{3} e_{3}+e_{3} q_{s} e_{3}\right)+\left(\frac{60}{\beta_{s \perp}^{3} \alpha_{s 1}^{\prime \prime}}-4\right) q_{s 3} e_{3} e_{3} e_{3}, \\
\mu_{s}=\frac{\rho_{s}}{2}\left(\frac{4}{\beta_{s \perp}^{2}}+\frac{1}{\beta_{s \perp} \beta_{s \|}}\right) \mathbf{I}+\frac{\rho_{s}}{2}\left[\frac{1}{\beta_{s \perp} \beta_{s \|}}+\frac{6}{\beta_{s \perp}}\left(\frac{q_{s 3}}{\rho_{s}}\right)^{4 / 3}+\frac{3}{\beta_{s \|}^{2}}-\frac{4}{\beta_{s \perp}^{2}}\right] e_{3} e_{3} \\
+\frac{1}{\beta_{s \perp} \alpha_{s 1}^{\prime \prime}}\left[24\left(\frac{q_{s 3}}{\rho_{s}}\right)^{5 / 3}+\frac{18}{\beta_{s \|}} \frac{q_{s 3}}{\rho_{s}}+\frac{8}{\beta_{s \perp}} \frac{q_{s 3}}{\rho_{s}}\right]\left(q_{s} e_{3}+e_{3} q_{s}-2 q_{s 3} e_{3} e_{3}\right) .
\end{gathered}
$$

With these closure assumptions, we can determine the left-hand side of the transport equations. The continuity equation (see eq. [9]) is the same as for the 16 moment approximation.

Momentum:

$$
\begin{aligned}
\rho_{s} \frac{D_{s} u_{s}}{D t}+\nabla_{\perp}\left(p_{s \perp}\right)+\nabla_{\|}\left(p_{s \|}\right)+e_{3}^{\top} \cdot\left[\nabla_{\|}\left(\frac{4 q_{s 3} q_{s}}{\rho_{s} \beta_{s \perp} \alpha_{s 1}^{\prime \prime}}\right)\right]+ & {\left[\nabla \cdot\left(\frac{4 q_{s 3} q_{s}}{\rho_{s} \beta_{s \perp} \alpha_{s 1}^{\prime \prime}}\right)\right] e_{3} } \\
& -\left[\nabla_{\|} \cdot\left(\frac{8 q_{s 3} q_{s}}{\rho_{s} \beta_{s \perp} \alpha_{s 1}^{\prime \prime}}\right)\right] e_{3}-\rho_{s} \boldsymbol{G}-n_{s} e_{s}\left(\boldsymbol{E}+\frac{1}{c} \boldsymbol{u}_{s} \times \boldsymbol{B}\right)=\frac{\delta \mathbf{M}_{s}}{\delta t},
\end{aligned}
$$

Parallel energy:

$$
\frac{D_{s} p_{s \|}}{D t}+\nabla_{\perp} \cdot\left(\frac{16 q_{s}}{\beta_{s \perp}^{3} \alpha_{s 1}^{\prime \prime}}\right)+\nabla \cdot\left(2 q_{s}\right)+\frac{2 \rho_{s}}{\beta_{s \|}}\left(\nabla \cdot u_{s \|}\right)+\frac{8 q_{s 3}}{\rho_{s} \beta_{s \perp} \alpha_{s 1}^{\prime \prime}}\left(\nabla_{\perp} u_{s \|}\right)^{\top}: q_{s} e_{3}+p_{s \|} \nabla \cdot u_{s}=\frac{\delta p_{s \|}}{\delta t},
$$

Perpendicular energy:

$$
\frac{D_{s} p_{s \perp}}{D t}+\nabla_{\perp} \cdot\left(\frac{8 \boldsymbol{q}_{s}}{\beta_{s \perp}^{3} \alpha_{s 1}^{\prime \prime}}\right)+p_{s \perp} \nabla \cdot u_{s \perp}+p_{s \perp} \nabla \cdot u_{s}+\frac{4 q_{s 3}}{\rho_{s} \beta_{s \perp} \alpha_{s 1}^{\prime \prime}}\left(\nabla_{\|} \boldsymbol{u}_{s \perp}\right)^{\top}: \boldsymbol{q}_{s} \boldsymbol{e}_{3}=\frac{\delta p_{s \perp}}{\delta t},
$$

Heat flux:

$$
\begin{aligned}
\frac{D_{s} \boldsymbol{q}_{s}}{D t}+ & {\left[\frac{D_{s} \boldsymbol{u}_{s}}{D t}-\boldsymbol{G}-\frac{e_{s}}{m_{s}}\left(\boldsymbol{E}+\frac{1}{c} \boldsymbol{u}_{s} \times \boldsymbol{B}\right)\right] \cdot\left[\left(2 p_{s \perp}+\frac{p_{s \|}}{2}\right) \mathbf{I}+\left(p_{s \|}-p_{s \perp}\right) \boldsymbol{e}_{3} \boldsymbol{e}_{3}\right.} \\
& \left.+\frac{4 q_{s 3}}{\rho_{s} \beta_{s \perp} \alpha_{s 1}^{\prime \prime}}\left(\boldsymbol{q}_{s} \boldsymbol{e}_{3}+\boldsymbol{e}_{3} \boldsymbol{q}_{s}-2 q_{s 3} \boldsymbol{e}_{3} \boldsymbol{e}_{3}\right)\right]+\nabla\left(\frac{2 \rho_{s}}{\beta_{s \perp}^{2}}+\frac{\rho_{s}}{2 \beta_{s \perp} \beta_{s \|}}\right)+\nabla_{\|}\left\{\frac{\rho_{s}}{2 \beta_{s \perp} \beta_{s \|}}+\frac{\rho_{s}}{2}\left[6\left(\frac{q_{s 3}}{\rho_{s}}\right)^{4 / 3}+\frac{3}{\beta_{s \|}^{2}}\right]-\frac{2 \rho_{s}}{\left.\beta_{s \perp}^{2}\right\}}\right. \\
& +\left(\nabla_{\|}\left\{\frac{1}{\alpha_{s 1}^{\prime \prime} \beta_{s \perp}}\left[24\left(\frac{q_{s 3}}{\rho_{s}}\right)^{5 / 3}+\frac{18}{\beta_{s \|}} \frac{q_{s 3}}{\rho_{s}}+\frac{8}{\beta_{s \perp}} \frac{q_{s 3}}{\rho_{s}}\right] \boldsymbol{q}_{s \perp}\right\}\right)^{\top} \cdot \boldsymbol{e}_{3}+\nabla \cdot\left\{\frac{1}{\alpha_{s 1}^{\prime \prime} \beta_{s \perp}}\left[24\left(\frac{q_{s}}{\rho_{s}}\right)^{5 / 3}+\frac{18}{\beta_{s \|}} \frac{q_{s 3}}{\rho_{s}}+\frac{8}{\beta_{s_{\perp}}} \frac{q_{s 3}}{\rho_{s}}\right] \boldsymbol{q}_{s \perp}\right\} \boldsymbol{e}_{3} \\
& +\frac{4 p_{s \perp}^{3}}{\alpha_{s 1}^{\prime \prime} \rho_{s}^{3}}\left[\nabla \boldsymbol{u}_{s} \cdot \boldsymbol{q}_{s \perp}+\left(\nabla \boldsymbol{u}_{s}\right)^{\top} \cdot \boldsymbol{q}_{s \perp}+\left(\nabla \cdot \boldsymbol{u}_{s}\right) \boldsymbol{q}_{s \perp}\right]+2\left(1-\frac{10}{\beta_{s \perp}^{3} \alpha_{s 1}^{\prime \prime}}\right)\left[\left(\nabla_{\|} \boldsymbol{u}_{s}\right)^{\top} \cdot \boldsymbol{q}_{s \perp}+\nabla_{\|} \boldsymbol{u}_{s} \cdot \boldsymbol{q}_{s \perp}+\left(\nabla_{\|} \cdot \boldsymbol{u}_{\mathbf{s}_{\|}}\right) \boldsymbol{q}_{s \perp}\right] \\
& +2\left(\nabla_{\|} \cdot \boldsymbol{u}_{s \|}\right) \boldsymbol{q}_{s \|}+\boldsymbol{q}_{s} \cdot \nabla \boldsymbol{u}_{s}+\boldsymbol{q}_{s} \boldsymbol{\nabla} \cdot \boldsymbol{u}_{s}-\frac{e_{s}}{m_{s} c}\left(\boldsymbol{q}_{s} \times \boldsymbol{B}\right)=\frac{\delta \boldsymbol{q}_{s}}{\delta t} .
\end{aligned}
$$

\section{REFERENCES}

Abramovitz, M., \& Stegun, I. A. 1964, Handbook of Mathematical Functions (New York: Dover)

Barghouthy, I. A., Barakat, A. R., \& Schunk, R. W. 1993, J. Geophys. Res., 98, 17583

Burgers, J. M. 1969, Flow Equations for Composite Gases (New York: Academic)

Chapman, S., \& Cowling, T. G. 1970, The Mathematical Theory of Nonuniform Gases (Cambridge: Cambridge Univ. Press)

Chew, G. F., Goldberger, M. L., \& Low, F. E. 1956, Proc. R. Soc. London A, 236, 112

Cordier, S. 1994a, Math. Models Methods Appl. Sci., 4, 625

- 1994b, Math. Models Methods Appl. Sci., 4, 647

Cordier, S., \& Girard, L. 1996, Planet. Space Sci., 44, 225

Demars, H. G., \& Schunk, R. W. 1979, J. Phys. D, 12, 1051

.1991, Planet. Space Sci., 39, 435

Dorelli, J. C., \& Scudder, J. D. 1996, EOS Trans., 77, 570
Feldman, W. C., Barraclough, B. L., Phillips, J. R., \& Wang, Y. M. 1996, A\&A, 316, 355

Grad, H. 1958, Principles of the Kinetic Theory of Gases (Handb. der Phys), 12

Groth, C. P. T., Gombosi, T. I., Roe, P. L., \& Brown, S. L. 1996, J. Fluid Mech., submitted

Habbal, S. R., Li, X., Esser, R., \& Hu, Y.-Q. 1996, EOS Trans., 77, 587

Hamburger, H. L. 1944, Am. J. Math., 66, 489

Hu, Y. Q., Esser, R., \& Habbal, S. R. 1997, J. Geophys. Res., 102, 14661

Jacques, S. A. 1977, ApJ, 215, 942

Kohl, J. L., Strachan, L., \& Gardner, L. D. 1996, ApJ, 465, L141

Leblanc, F., \& Hubert, D. 1997, ApJ, 483, 464 (Paper I)

Levermore, C. D. 1995, J. Stat. Phys., submitted

Levermore, C. D., Morokoff, W. J., \& Nadiga, B. T. 1995, Phys. Fluids, submitted

Marsch, E. 1982, in Solar Wind Five (Washington: NASA), 355 
Marsch, E., Mulhauser, K. H., Schwenn, R., Rosenbauer, H., Pillip, W., \& Neubauer, F. M. 1982, J. Geophys. Res., 87, 52

Mintzer, D. 1965, Phys. Fluids, 8, 1076

Palmadesso, P. J., Ganguli, S. B., \& Mitchell, H. G., Jr. 1988, Geophys. Monogr. Ser., 44, 133

Reed, M., \& Simon, B. 1975, Methods of Modern Mathematical Physics II: Fourier Analysis, Self-Adjointness (New York: Academic)
Robineau, A., Blelly, P.-L., \& Fontanari, J. 1996, J. Atmos. Terr. Phys., 58, 257

Spitzer, L., Jr. 1952, ApJ, 116, 299

Tanenbaum, B. S. 1967, Plasma Physics (New York: Mc Graw-Hill)

Withbroe, G. L. 1988, ApJ, 325, 442 